www.nature.com/jhg

\title{
Inheritance of polyalanine expansion mutation of PHOX2B in congenital central hypoventilation syndrome
}

\author{
Toru Meguro ${ }^{1}$, Yuki Yoshida ${ }^{1}$, Makiko Hayashi ${ }^{1}$, Kentaro Toyota ${ }^{1}$, Tesshu Otagiri ${ }^{1}$, Narutaka Mochizuki ${ }^{2}$, \\ Yumiko Kishikawa $^{1}$, Ayako Sasaki ${ }^{1}$ and Kiyoshi Hayasaka ${ }^{1}$
}

Congenital central hypoventilation syndrome (CCHS; MIM 209880) is caused mostly by dominant alanine expansion (most prevalent is 7-alanine expansion) mutations in PHOX2B. More than $90 \%$ of the alanine expansion mutations had been considered to be de novo due to unequal crossover during gametogenesis. However, a recent report stated that $25 \%$ of patients inherited the alanine-expanded allele from their parents with somatic mosaicism or constitutive mutation. We studied inheritance in 45 unrelated families, and found that one patient $(2 \%)$ inherited 5 -alanine expansion mutation from a parent with late-onset central hypoventilation syndrome and nine patients $(20 \%)$ inherited 5- to 7-alanine expansion mutation from apparently asymptomatic parents with somatic mosaicism. Analysis using a sensitive method would be recommended to all parents of CCHS proband due to high incidence of somatic mosaicism. The absence of an alanine-contracted allele (expected counterpart allele in unequal crossover) and the highest prevalence of 6-alanine expansion mutation in somatic mosaicism suggest that the somatic mosaicism is likely caused by a mechanism other than an unequal crossover, such as a replication mechanism.

Journal of Human Genetics (2012) 57, 335-337; doi:10.1038/jhg.2012.27; published online 22 March 2012

Keywords: congenital central hypoventilation syndrome; mosaic; PHOX2B; polyalanine

\section{INTRODUCTION}

Congenital central hypoventilation syndrome (CCHS; MIM 209880) is characterized by failure of the automatic control of breathing during sleep, and results from the dominant PHOX2B mutation. More than $90 \%$ of patients have polyalanine expansion mutations in the polyalanine tract of 20 residues, and less than $10 \%$ of patients have frameshift or point mutations. ${ }^{1-4}$ More than $90 \%$ of polyalanine expansion mutations have been considered to be de novo, and about $10 \%$ of the mutations were thought to have been inherited mostly from asymptomatic parents with somatic mosaicism and rarely from affected parents. ${ }^{4-6}$ Most recently, Bachetti et al. ${ }^{7}$ analyzed 52 parents using a sensitive method involving coupling amplification with carboxyfluorescein (FAM)-tagged primers and capillary electrophoresis, and found that 13 of the cases (25\%) inherited the expanded mutations from a parent with late-onset central hypoventilation syndrome (LOCHS) or asymptomatic parents with somatic mosaicism or constitutive mutation. Herein, we studied the inheritance of expanded mutation in 45 unrelated families, and found that one patient (2\%) inherited it from the mother with LO-CHS, and nine patients (20\%) inherited each mutation from apparently asymptomatic parent with somatic mosaicism.

\section{MATERIALS AND METHODS}

CCHS families analyzed

The ethics committee of the Yamagata University School of Medicine approved this study. After receiving written informed consent from the patients' families, peripheral blood was collected from family members for genomic DNA extraction. We studied 45 CCHS families with alanine expansion mutation. In all, 8 patients carried 5 -alanine, 15 patients carried 6 -alanine, 18 patients carried 7-alanine, 1 patient carried 11-alanine, 2 patients carried 12-alanine and 1 patient carried 13-alanine expansion mutations (Figures 1 and 2). One patient (case 79) was speculated to carry 7-alanine expansion mutation. This family had two deceased sibling patients complicated with Hirschsprung's disease, whose specimens were not available for genetic analysis. The parents cooperated with the study, and the father was identified as showing somatic mosaicism of 7-alanine expansion mutation (Table 1).

\begin{abstract}
Molecular analysis of PHOX2B
We screened the coding region of $P H O X 2 B$ by direct sequencing essentially according to the method described by Matera et al. ${ }^{8}$ For the analysis of somatic mosaicism of the family members of the patients carrying polyalanine expansion mutation, we amplified a 458-bp fragment or a 254-bp fragment including a polyalanine sequence in exon 3 from genomic DNA with primers 22F $5^{\prime}$-GCACTGACCCGGACAGCACT- $3^{\prime}$ and 3.3R 5'-TACCCGCTCGCCCA
\end{abstract}

${ }^{1}$ Department of Pediatrics, Yamagata University School of Medicine, Yamagata, Japan and ${ }^{2}$ Department of Neonatal Medicine, Osaka Medical Center and Research Institute for Maternal and Child Health, Izumi, Osaka, Japan

Correspondence: Professor K Hayasaka, Department of Pediatrics, Yamagata University School of Medicine, 2-2-2 lida-nishi, Yamagata 990-9585, Japan.

E-mail: hayasaka@med.id.yamagata-u.ac.jp

Received 11 December 2011; revised 22 February 2012; accepted 26 February 2012; published online 22 March 2012 
Phenotypic variability in the family of case 17 carrying 5-alanine expansion mutation

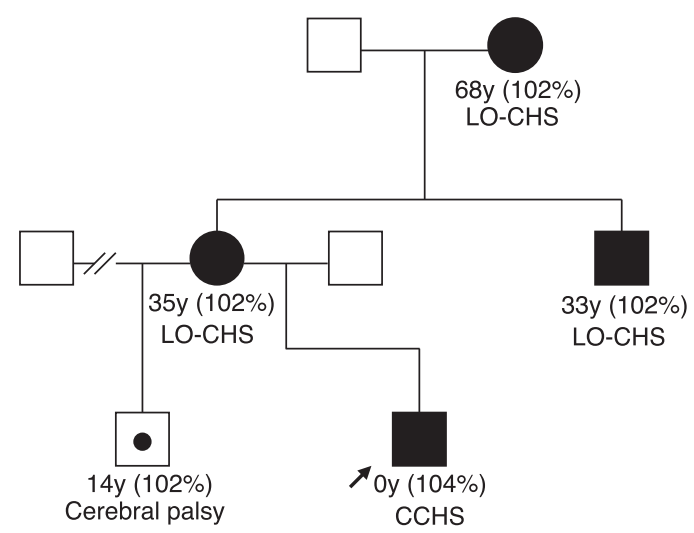

Figure 1 Phenotypic variability in the family of case 17 carrying 5-alanine expansion mutation. Filled squares and circles indicate affected males and females, respectively. Numbers under symbols and those in parentheses represent the age and percentage of mosaicism, respectively. The arrow indicates the proband. CCHS, congenital central hypoventilation syndrome; LO-CHS, late-onset central hypoventilation.

Number of alanine-expansion mutations in the studied patients and in the cases inherited from mosaic parents

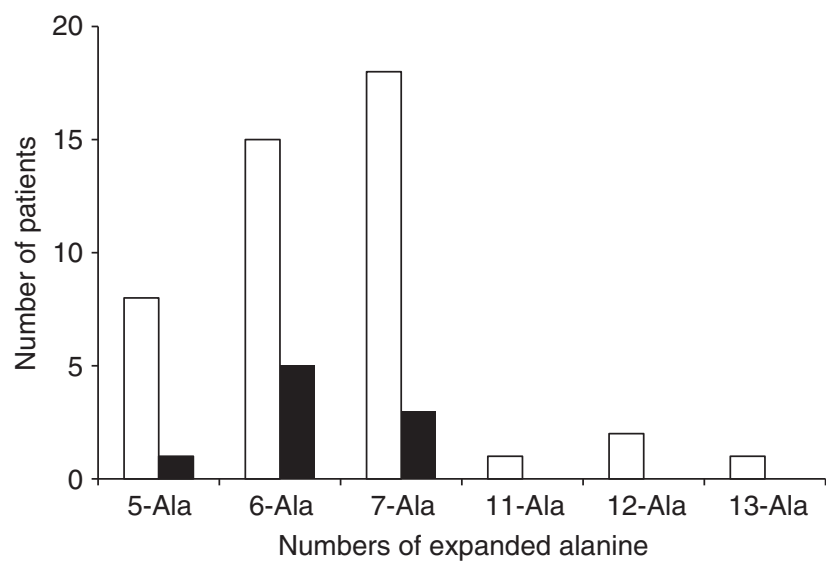

Figure 2 Number of alanine-expansion mutations in the studied patients and in the patients inherited from mosaic parents. Open and filled bars represent the studied patients and patients inherited from the mosaic parents, respectively.

CTCG-3' or with primers 22F $5^{\prime}$-GCACTGACCCGGACAGCACT- $3^{\prime}$ and 279R $5^{\prime}$-GAGCCCAGCCTTGTCCAGG- $3^{\prime}$; the forward probes were $5^{\prime}$ labeled with carboxyfluorescein (FAM) according to the method of Trochet et al. ${ }^{5,9}$ and Bachetti et al. ${ }^{7}$ We loaded the PCR product on a 3130 Capillary Sequencer (PE Applied Biosystems, Foster City, CA, USA), used ROX500 (PE Applied Biosystems) as a size marker, and analyzed somatic mosaicism using GeneMapper version 4.1 software (PE Applied Biosystems). The percentage of mutant alleles was estimated by dividing the area under the peak corresponding to the mutant allele (E) by the sum of wild type (W) and mutant peak areas $(\mathrm{E}+\mathrm{W})$. Mosaicism of 5-, 6- and 7-alanine expansion mutations and mosaicism of 11-, 12- and 13-alanine expansion mutations were detected using primers with $22 \mathrm{~F}$ and $3.3 \mathrm{R}$, and using primers with $22 \mathrm{~F}$ and $3.3 \mathrm{R}$, respectively. To validate the technique and correct the percentage of mutant alleles, DNA solutions containing 1.25, 2.5, 3.75, 5, 12.5, 25, 32.5 and $50 \%$ of 5-, 6-, 7- and 13-alanine expansion mutations were prepared by mixing genomic DNA from controls and patients, of which the mixing ratio was
Table 1 Rate of mosaicism in the parents with somatic mosaicism

\begin{tabular}{lcccccc}
\hline $\begin{array}{l}\text { Case } \\
\text { number }\end{array}$ & Sex & $\begin{array}{c}\text { Parents } \\
\text { (mosaicism) }\end{array}$ & $\begin{array}{c}\text { Expanded } \\
\text { alanine }\end{array}$ & $\begin{array}{c}\text { Affected } \\
\text { siblings }\end{array}$ & $\begin{array}{c}\text { E/E }+W \\
\text { (observed) }\end{array}$ & $\begin{array}{c}\text { (\%) } \\
\text { (calculated) }\end{array}$ \\
\hline Case 3 & Female & Mother & 6-Alanine & - & 0.251 & 51 \\
Case 41 & Female & Father & 6-Alanine & - & 0.025 & 5 \\
Case 46 & Male & Father & 6-Alanine & - & 0.043 & 9 \\
Case 55 & Female & Father & 6-Alanine & - & 0.023 & 5 \\
Case 68 & Male & Mother & 5-Alanine & + & 0.306 & 62 \\
Case 79 & Male & Father & 7-Alanine & + & 0.040 & 8 \\
Case 84 & Male & Mother & 6-Alanine & + & 0.269 & 55 \\
Case 85 & Male & Father & 7-Alanine & - & 0.028 & 6 \\
Case 97 & Female & Father & 7-Alanine & - & 0.280 & 58
\end{tabular}

The percentage of mutant alleles was estimated by dividing the area under the peak corresponding to the mutant allele (E) by the sum of wild type (W) and mutant peak areas $(\mathrm{E}+\mathrm{W})$.

a-alanine expansion was speculated from his father's data.

determined by the content of the template for the glyceraldehyde-3-phosphate dehydrogenase gene spanning from nucleotide 804 to nucleotide 903 (NM_002046.2) estimated by real-time PCR. ${ }^{10}$ Linear regression between the amounts of different alanine-expanded alleles and expected mosaicism levels were estimated with the degree of correlation measured by the $\mathrm{R}^{2}$ coefficient. The equation of the regression line and $\mathrm{R}^{2}$ coefficient for 5-, 6-, 7- and 13alanine expansion mutation were $Y=0.49 \mathrm{X} \quad\left(R^{2}=0.9948\right), \quad Y=0.49 \mathrm{X}$ $\left(R^{2}=0.9998\right) . \quad Y=0.48 \mathrm{X} \quad\left(R^{2}=0.9986\right) \quad$ and $\quad Y=0.46 \mathrm{X} \quad\left(R^{2}=0.9977\right)$, respectively.

To confirm the mosaicism of the parents detected by fragment analysis, a PCR fragment was subcloned into the TA cloning vector and the sequence of expanded clones was determined.

\section{RESULTS}

Among 45 CCHS families, we found that one patient inherited 5 -alanine expansion mutation from a parent affected with LO-CHS (Figure 1), and nine patients inherited alanine expansion mutation from apparently asymptomatic parents with somatic mosaicism (Table 1). One patient inherited 5-alanine, five patients inherited 6-alanine, and three patients inherited 7-alanine expansion mutations from the parents with somatic mosaicism (Table 1, Figure 2). The percentage of mosaicism in each parent ranged from $5-62 \%$ and three patients had affected siblings. Six patients inherited expansion mutation from their fathers and three patients inherited it from their mothers.

Case 17, who was heterozygous for 5-alanine expansion mutation, presented with respiratory symptoms in the neonatal period, and his mother and grandmother were diagnosed with LO-CHS by polysomnography at the age of 35 and 68 years, respectively, following the diagnosis of the proband (Figure 1). His uncle had cardiac failure at the age of 31 years, and was confirmed to have LO-CHS by polysomnography. His half-brother, aged 14 years, was affected with cerebral palsy due to an unknown cause, but had no sleep apnea on polysomnography. The proband, half-brother, mother, uncle and grandmother were heterozygotes for 5-alanine expansion mutation (Figure 1).

\section{DISCUSSION}

Our study of 45 CCHS families revealed that one case (2\%) inherited 5 -alanine expansion mutation from the parent with LO-CHS (Figure 1) and nine cases (20\%) inherited 5- to 7-alanine expansion mutation from the parents with somatic mosaicism (Table 1). Most CCHS patients carry 5- to 13-alanine expansion mutations in 
PHOX2B, and more than $90 \%$ of its mutations had been considered to be de novo. ${ }^{4-6}$ However, Bachetti et al. ${ }^{7}$ analyzed 52 parents using a sensitive method, and found that 13 cases (25\%) inherited the expanded mutations from their parents: 1 case inherited 5-alanine expansion mutation from a parent with LO-CHS, 2 cases inherited 5-alanine expansion mutation from asymptomatic parents with constitutive mutation, and 10 cases (five cases with 6-alanine, three cases with 7-alanine, 1 case with 9-alanine and 1 case with 13 -alanine expansion mutation) from the parents with somatic mosaicism. As shown in Table 1, the level of mosaicism of each parent ranged from $5-62 \%$. Three patients had affected siblings. The family of case 79 had two deceased sibling patients complicated with Hirschsprung's disease, whose specimens were not available for genetic analysis. Considering the recurrent mutation in this family, we analyzed the parents for genetic mosaicism, and detected the somatic mosaicism of 7-alanine expansion mutation in the father. The expanded mutation was definitely transmitted to the descendants even with the low level of mosaicism (5\%) in the somatic genomes of the parent. The proportion of mutant alleles in somatic mosaicism may not reflect the proportion in germline mosaicism. Our study confirmed a highfrequency inheritance of alanine expansion mutation from mosaic parents and we would recommend testing all parents of CCHS proband with a sensitive fragment analysis. ${ }^{7,10}$

The symptoms of the patients likely depend on the length of the expanded alanine. The individuals carrying 5-alanine expansion mutations are known to present central hypoventilation in the neonatal period or in a later period (LO-CHS), or are asymptomatic due to incomplete penetrance. ${ }^{11}$ Therefore, the patients carrying 5-alanine expansion mutation would inherit the mutation from asymptomatic parents (parents with constitutive mutation or mosaicism) or parents affected with LO-CHS. Case 17 was a patient showing neonatal onset, and his mother and grandmother were diagnosed with LO-CHS by polysomnography at the age of 35 and 68 years, respectively, following the diagnosis of the proband (Figure 1). As for the symptoms in somatic mosaicism, nearly all individuals with somatic mosaicism were asymptomatic. However, one case mosaic for 8-alanine expansion mutation developed central hypoventilation in his forties. ${ }^{12}$ The mosaic parents may require polysomnographic examination.

We previously reported that de novo alanine expansion mutation was of paternal origin and possibly caused by unequal sister chromatid exchange during spermatogenesis. ${ }^{13-15}$ However, a significantly high incidence of the mutation inherited from mosaic parents may raise the question of whether de novo polyalanine expansions are due to unequal crossover in the germline. No alanine-contracted-counterpart allele (expected counterpart allele in unequal crossover) was detected in individuals showing somatic mosaicism. In contrast, polymorphic alanine-contracted alleles were frequently detected in healthy individuals. ${ }^{1,3,4,16}$ It is also interesting to note that the most prevalent alanine-expanded allele is different between whole CCHS patients and the patients inherited from mosaic parents (Figure 2). Seven- and six-alanine-expanded alleles are most prevalent in whole CCHS patients and the patients inherited from mosaic parents, respectively (Figure 2). ${ }^{5-7,10}$ Therefore, alanine expansion mutation in somatic mosaicism is not likely caused by an unequal crossover, but by a replication mechanism such as a model of repeat instability generated during the replication fork stalling and restart within the repetitive run. ${ }^{17}$ Even though about $25 \%$ of the alanine expansion mutations would be transmitted from the parents with mosaicism or constitutive mutation, a major part (about $75 \%$ ) of mutation is likely de novo.

In conclusion, nearly $25 \%$ of CCHS patients carrying polyalanine expansion mutation inherit the mutation from parents with somatic mosaicism or constitutive mutation, and familial analysis using a sensitive method is needed for effective genetic counseling and the study of the alanine expansion mechanism.

\section{ACKNOWLEDGEMENTS}

We thank the patients and their families for their cooperation. This work was supported by a Grant-in-Aid for Scientific Research from the Ministry of Education, Science, Culture and Sports of Japan.

1 Amiel, J., Laudier, B., Attie-Bitach, T., Trang, H., de Pontual, L. \& Gener, B. et al. Polyalanine expansion and frameshift mutations of the paired-like homeobox gene PHOX2B in congenital central hypoventilation syndrome. Nat. Genet. 33, 459-461 (2003).

2 Sasaki, A., Kanai, M., Kijima, K., Akaba, K., Hashimoto, M. \& Hasegawa, H. et al. Molecular analysis of congenital central hypoventilation syndrome. Hum. Genet. 114, 22-26 (2003).

3 Matera, I., Bachetti, T., Puppo, F., Di Duca, M., Morandi, F. \& Casiraghi, G. M. et al. PHOX2B mutations and polyalanine expansions correlate with the severity of the respiratory phenotype and associated symptoms in both congenital and late onset central hypoventilation syndrome. J. Med. Genet. 41, 373-380 (2004).

4 Weese-Mayer, D. E., Berry-Kravis, E. M., Zhou, L., Maher, B. S., Silvestri, J. M. \& Curran, M. E. et al. Idiopathic congenital central hypoventilation syndrome: analysis of genespertinent to early autonomic nervous system embryologic development and identification of mutations in PHOX2b. Am. J. Med. Genet. A 123A, 267-278 (2003).

5 Trochet, D., O'Brien, L. M., Gozal, D., Trang, H., Nordenskjold, A. \& Laudier, B. et al. $\mathrm{PHOX} 2 \mathrm{~B}$ genotype allows for prediction of tumor risk in congenital central hypoventilation syndrome. Am. J. Hum. Genet. 76, 421-426 (2005).

6 Berry-Kravis, E. M., Zhou, L., Rand, C. M. \& Weese-Mayer, D. E. Congenital central hypoventilation syndrome: PHOX2B mutations and phenotype. Am. J. Respir. Crit. CareMed. 174, 1139-1144 (2006).

7 Bachetti, T., Parodi, S., Di Duca, M., Santamaria, G., Ravazzolo, R. \& Ceccherini, I. Low amounts of PHOX2B expanded alleles in asymptomatic parents suggest unsuspected recurrence risk in congenital central hypoventilation syndrome. J. Mol. Med. (Berl). 89, 505-513 (2011).

8 Matera, I., Bachetti, T., Puppo, F. Di Duca, M. Morandi, F. \& Casiraghi, G. M. et al. PHOX2B mutations and polyalanine expansions correlate with the severity of the respiratory phenotype and associated symptoms in both congenital and late onset central hypoventilation syndrome. J. Med. Genet. 41, 373-380 (2004).

9 Trochet, D., Hong, S. J., Lim, J. K., Brunet, J. F., Munnich, A. \& Kim, K. S. et al. Molecular consequences of PHOX2B missense, frameshift and alanine expansion mutations leading to autonomic dysfunction. Hum. Mol. Genet. 14, 3697-3708 (2005).

10 Jennings, L. J., Yu, M., Zhou, L., Rand, C. M., Berry-Kravis, E. M. \& Weese-Mayer, D. E. Comparison of PHOX2B testing methods in the diagnosis of congenital central hypoventilation syndrome and mosaic carriers. Diagn. Mol. Pathol. 19, 224-231 (2010).

11 Weese-Mayer, D. E., Berry-Kravis, E. M., Ceccherini, I., Keens, T. G., Dariu, A. \& Loghmanee, D. A. et al. An oficial ATS clinical policy statement: Congenital central hypoventilation syndrome. Genetic basis, diagnosis, and management. Am. J. Respir. Crit. Care Med. 181, 626-644 (2010).

12 Trochet, D., de Pontual, L., Straus, C., Gozal, D., Trang, H. \& Landrieu, P. et al. PHOX2B germline and somatic mutations in late-onset central hypoventilation syndrome. Am. J. Respir. Crit. Care Med. 177, 906-911 (2008).

13 Warren, S. T. Polyalanine expansion in synpolydactyly might result from unequal crossing-over of HOXD13. Science 275, 408-409 (1997).

14 Arai, H., Otagiri, T., Sasaki, A., Hashimoto, T., Umetsu, K. \& Tokunaga, K. et al. De novo polyalanine expansion of PHOX2B in congenital central hypoventilation syndrome: unequal sister chromatid exchange during paternal gametogenesis. J. Hum. Genet 52, 921-925 (2007).

15 Arai, H., Otagiri, T., Sasaki, A., Umetsu, K. \& Hayasaka, K. Polyalanine expansion of PHOX2B in congenital central hypoventilation syndrome: $r s 17884724: A>C$ is associated with 7-alanine expansion. J. Hum. Genet 55, 4-7 (2009).

16 Horiuchi, H., Sasaki, A., Osawa, M., Kijima., K., Ino, Y. \& Matoba, R. et al. Sensitive detection of polyalanine expansions in $\mathrm{PHOX} 2 \mathrm{~B}$ by polymerase chain reaction using bisulfite-converted DNA. J. Mol. Diagn. 7, 638-640 (2005).

17 Mirkin, S. M. Expandable DNA repeats and human disease. Nature 447, 932-940 (2007). 